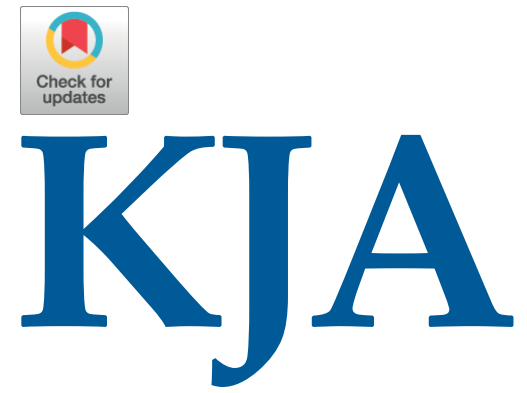

Korean Journal of Anesthesiology

\title{
Recommendations for anesthesia in patients suspected of COVID-19 Coronavirus infection
}

\author{
Hyun Joo Kim ${ }^{1}$, Justin Sangwook Ko ${ }^{2}$, Tae-Yop Kim ${ }^{3}$, Scientific \\ Committee of the Korean Society of Anesthesiologists
}

\section{Invited Editorial}

Korean J Anesthesiol 2020;73(2):89-91

https://doi.org/10.4097/kja.20110

pISSN 2005-6419 • elSSN 2005-7563

Received: March 12, 2020

Accepted: March 15, 2020

Corresponding author:

Tae-Yop Kim, M.D., Ph.D.

Department of Anesthesiology and Pain

Medicine, Konkuk University Medical Center,

Konkuk University School of Medicine, 120-1

Neungdong-ro, Gwangjin-gu, Seoul 05030,

Korea

Tel: +82-2-2030-5445

Fax: +82-2-2030-5449

Email: taeyop@gmail.com

ORCID: https://orcid.org/0000-0003-0806-8969
Department of Anesthesiology and Pain Medicine, ${ }^{1}$ Anesthesia and Pain Research Institute, Yonsei University College of Medicine, ${ }^{2}$ Samsung Medical Center, Sungkyunkwan University School of Medicine, ${ }^{3}$ Konkuk University Medical Center, Konkuk University School of Medicine, Seoul, Korea

The following guidelines are based on recommendations from the Anesthesia Patient Safety Foundation (APSF) for working with patients who have COVID-19 [1] and the other literature [2-5], and can be modified and adapted to the circumstances of each institution or hospital. When managing patients with confirmed or suspected COVID-19 infection, it is of utmost importance to protect health care workers from infection. All medical personnel must be provided with personal protective equipment (PPE) to prevent droplet and contact infections.

Medical staff and institutions should establish procedural protocols for donning and removing PPE. Prior to patient treatment, staff must identify and review the in-hospital procedural protocols, and plan ahead for patient transfer, anesthesia work environment, and anesthesia methods.

\section{Patient transport and operating room managing plan}

- Do not allow patients to stay in the holding area. Treatment should be carried out in a pre-allocated negative pressure operating room. Warning signs for COVID-19 infection should be posted in front of the operating room to minimize staff exposure.

- Patients should not stay in the recovery room or postoperative care unit. After complete recovery in the operating room, the patient should be transferred into a negative pressure room in a ward or intensive care unit (ICU).

- Endotracheal intubation, tube exchange, and extubation are high-risk procedures that can expose healthcare workers to respiratory droplets of the virus. These procedures must be carried out in a location where negative pressure is applied such as negative pressure room, and special care should be taken.

- Depending on the clinical situation, performing an endotracheal intubation early in a negative pressure room in a ward or ICU, rather than in an operating room, should be considered.

- If it is determined that the degree of negative pressure in the environment is not sufficient, the additional application of a portable high-efficiency particulate air filter should be considered.

- A highly efficient hydrophobic filter should be placed between the endotracheal tube and the reservoir bag, to prevent atmospheric contamination by respiratory droplets during patient transfers. 


\section{Anesthesia Process}

\section{Preparation of manpower}

- Assign the most experienced anesthesia professionals to practitioners performing endotracheal intubations. Inexperienced trainees should not perform endotracheal intubation for training purposes.

- Assign experienced assistants who can perform techniques such as cricoid pressure when performing rapid sequence induction (RSI).

- Consider allowing anesthesia teams to be replaced at least every 2 hours, to prevent fatigue.

\section{Preparation before anesthesia and use of personal protective equipment}

- Allow sufficient time for all staff involved to don PPE. It may take more than 5 minutes to properly wear PPE.

- Early planning and implementation of endotracheal intubation should be considered. In the event of an unexpected emergency endotracheal intubation, PPE cannot be adequately donned; therefore, early implementation should be performed when possible.

- Protective coverall/body suits, N95 masks, disposable goggles/ face shields, disposable shoe covers, and disposable gloves must be worn. Use the double glove technique on both hands to reduce contact.

- A powered air-purifying respirator should be worn by healthcare workers who are involved in endotracheal intubation or extubation processes.

\section{Selection of intubation technique}

- Awake fiberoptic intubation should not be performed unless it is a necessary indication. Spraying local anesthetics can aerosolize the virus, and should be avoided.

- Consider using video laryngoscopes to increase the likelihood of successful endotracheal intubation.

- Consider using disposable devices for intubation.

\section{Endotracheal intubation}

- A high-efficiency hydrophobic filter must be applied between the face mask and the breathing circuit, or between the face mask and the reservoir bag.

- Preoxygenation for 5 minutes with $100 \%$ oxygen should be performed.

- RSI should be performed to limit procedures such as manual ventilation, which can spread aerosolized virus into the room.

- The method of RSI can be modified to suit the clinical situation. If manual ventilation is required, a small tidal volume may be considered, or a supraglottic airway may be inserted to provide ventilation instead of manual ventilation using a face mask.

- Do not use high-flow oxygen, such as high-flow nasal cannula devices, as these can aerosolize the virus.

\section{Equipment management after endotracheal intubation}

- All used airway equipment should be placed in double ziplocked plastic bags and removed for disposal or disinfection.

- Used laryngoscopes should be sealed in double zip-locked plastic bags as soon as the endotracheal intubation is complete, to prevent further contamination of the surroundings.

- End-tidal carbon dioxide sample lines and traps should be replaced.

- Take care to avoid contaminating various instruments in the operating room, such as stethoscopes, pens, and telephones.

\section{Undressing and hand washing after endotracheal intubation}

- Consider preparing additional isolation rooms as contaminated areas next to the operating room to remove and dispose of PPE in accordance with protocol. If it is difficult to obtain an additional isolation room, use the space inside or immediately outside the operating room to remove PPE according to the protocol.

- Wash your hands after removing PPE.

- Avoid body contact, including touching your hair or face, until hands are washed.

\section{Acknowledgments}

We thank the members of Scientific Committee in the Korean Society of Anesthesiologists; Eun-Ho Lee (University of Ulsan College of Medicine, Seoul), Hyungseok Seo (Kyung Hee University College of Medicine, Seoul), Sun Young Park (Soonchunhyang University College of Medicine, Seoul), Jin-Tae Kim (Seoul National University College of Medicine, Seoul), Seo-kyung Shin (Yonsei University College of Medicine, Seoul), Jinseok Yeo (Kyungpook National University School of Medicine, Daegu), Dong-Kyu Lee (Korea University College of Medicine, Seoul), 
Jong-Hwan Lee (Sungkyunkwan University School of Medicine, Seoul), WooSuk Chung (Chungnam National University College of Medicine, Daejeon), Geun Joo Choi (Chung-Ang University College of Medicine, Seoul), Jae Hee Woo (Ewha Womans University College of Medicine, Seoul), Ji Su Jang (Hallym University College of Medicine, Chuncheon), Ah-Reum Cho (Pusan National University College of Medicine, Busan).

\section{Conflicts of Interest}

No potential conflict of interest relevant to this article was reported.

\section{Author Contributions}

Hyun Joo Kim (Investigation; Project administration; Supervision; Validation; Writing - original draft; Writing - review \& editing)

Justin Sangwook Ko (Validation; Writing - original draft; Writing - review \& editing)

Tae-Yop Kim (Conceptualization; Project administration; Resources; Writing - original draft; Writing - review \& editing)

\section{ORCID}

Hyun Joo Kim, https://orcid.org/0000-0003-1963-8955
Justin Sangwook Ko, https://orcid.org/0000-0003-3155-0550

Tae-Yop Kim, https://orcid.org/0000-0003-0806-8969

\section{References}

1. Liana Z, Nadav L, Desire K, Mike A, Satya KR. Perioperative Considerations for the 2019 Novel Coronavirus (COVID-19) [Internet]. Rochester (MN): Anesthesia patient safety foundation; 2020 Feb 12 [cited 2020 Mar 12]. Available from https:// www.apsf.org/news-updates/perioperative-considerationsfor-the-2019-novel-coronavirus-covid-19/.

2. Kamming D, Gardam M, Chung F. Anaesthesia and SARS. Br J Anaesth 2003; 90: 715-8.

3. Peng PW, Wong DT, Bevan D, Gardam M. Infection control and anesthesia: lessons learned from the Toronto SARS outbreak. Can J Anaesth 2003; 50: 989-97.

4. Cheung JC, Ho LT, Cheng JV, Cham EYK, Lam KN. Staff safety during emergency airway management for COVID-19 in Hong Kong. Lancet Respir Med 2020. Advance access published on Feb 24, 2020. doi: 10.1016/S2213-2600(20)30084-9.

5. Caputo KM, Byrick R, Chapman MG, Orser BJ, Orser BA. Intubation of SARS patients: infection and perspectives of healthcare workers. Can J Anaesth 2006; 53: 122-9. 